\title{
Combining Grounded Theory (GT) and Structural Equation Modelling (SEM) to analyze indoor soundscape in historical spaces
}

\author{
Volkan Acun*, Semiha Yilmazer \\ Bilkent University, Faculty of Art, Design and Architecture, Department of Interior Architecture and Environmental Design, Bilkent, Ankara 06800, Turkey
}

\section{A R T I C L E I N F O}

\section{Article history:}

Received 14 February 2019

Received in revised form 12 June 2019

Accepted 14 June 2019

Available online 27 June 2019

\section{Keywords:}

Indoor soundscape

Grounded theory

Structural equation modelling

\begin{abstract}
A B S T R A C T
This paper reports the findings of indoor soundscape research that was conducted in a historical museum setting. The study took place in the historic caravanserai of Cengelhan, which is currently a part of the Rahmi Koc Museum. A combination of qualitative and quantitative research methods were used to capture visitors' subjective museum experience and understand the role of the sound environment. In this sense, the research is divided into two parts: Grounded Theory (GT) and Structural Equation Modelling (SEM). For the qualitative part, semi-structured interviews were held as part of GT, to create a conceptual framework that reflects visitors' perception of the indoor soundscape of the museum. Hypotheses were derived from this conceptual framework based on the patterns between the categories of the conceptual model. In the second part, this model was tested for statistical significance and path correlations through the SEM method. The core categories of the conceptual framework are similar to the previous research, mainly the categories of Context, Interpretation, and Outcomes. The SEM analysis showed that categories of Expectation and Preference were not significantly associated with the Built or Sound Environment. Findings showed that a positive interpretation of the sound environment could create a livable environment with a unique atmosphere which would increase visitors' interaction with the museum and positively affect their experience.
\end{abstract}

(c) 2019 Elsevier Ltd. All rights reserved.

\section{Introduction}

Museums are environments which are dedicated to education, learning and providing recreation to visitors. They serve society by preserving, maintaining and exhibiting human heritage [1]. The experience undergone in a museum used to be a passive one. Since the mid-twentieth century, however, the developments in technology are changing this experience and making it more and more active [2]. Museums are searching for new ways of enhancing knowledge and encouraging visitors to engage more actively with the displayed objects.

Experience is influenced by interaction with different contexts. It involves various elements and provides emotional, physical, intellectual and spiritual mixed feelings [3]. Historic environments, on the other hand, have their own spatiality and a variety of different socio-cultural functions that characterize them. Preservation of historic spaces can help transfer the historical information, that is contained in the space, to its visitors and increase their interaction

\footnotetext{
* Corresponding author.

E-mail addresses: volkan.acun@bilkent.edu.tr (V. Acun), semiha@bilkent.edu.tr (S. Yilmazer).
}

with the environment [4]. One possible way to increase this interaction can be achieved by using sound as a design element. But we first need to understand the role of sound in a museum environment and how it is perceived by the visitors, to evaluate if it would be worth to be implemented.

Soundscape is an approach that is concerned with the perception, interpretation and the meanings associated with the sound, rather than the sound energy itself. According to ISO 12913-1, the soundscape is defined as "the acoustic environment perceived or experienced and/or understood by a person or people, in context" [5].

Most recently, ISO published the second part of the soundscape standard, the ISO 12913-2:2018, which focuses on data collection and reporting requirements [6]. While suggesting minimum requirements for reporting the soundscape findings, it also suggests three examples of data collection. The first method is a quantitative method that uses a questionnaire survey, while the second one also uses quantitative means with an emphasis on the soundwalks. The third method is a qualitative method, which uses narrative interviews and provides a comprehensive list of structured questions that can be used during interviews. However, in this study, the data collection for Grounded Theory (GT) and the 
questionnaire survey was done prior to the publication of the ISO 12913-2, therefore, while some parts of our data collection tools are similar, some are not compatible with this standard.

Aletta et al. [7] made a comprehensive revision of the soundscape descriptors and data collection methods that are used in soundscape research so far. According to this, there are eight soundscape descriptors: noise annoyance, pleasantness, quietness or tranquillity, music-likeness, perceived affective quality, restrictiveness, soundscape quality, and appropriateness. Data regarding this descriptors can be collected with various tools: questionnaires [8,9], semantic scales [10-12], interviews protocols [8,13-17], binaural recordings and/or psychoacoustic measurements [18].

While the urban soundscapes have been investigated over the decades, the indoor soundscape studies begun more recently. So far, indoor soundscape research covered a variety of different indoor soundscapes [19], such as hospitals [8], open-offices [16], education facilities [10,20], care facilities [21], libraries [22-24], worship places [15], transportation hubs [25]. One of the first examples of indoor soundscape research has been conducted by Mackrill et al., when they used qualitative methods to capture the subjective soundscape perception of a hospital ward. Their findings revealed that patients could cope with the soundscape by accepting and habituating to it [8]. Cankaya and Yilmazer researched high school classrooms and found out that irrelevant sounds are perceived negatively [20]. Acun and Yilmazer developed a conceptual framework to capture individuals' perception of the soundscape in open offices [16]. They also observed that individuals develop methods to cope with unsatisfactory soundscapes. Another GT research conducted by Yilmazer and Acun investigated the soundscape of the historic Hacı Bayram Mosque of Ankara, which showed the associations between the soundscape elements, spatial function and place identity [15]. Another indoor soundscape research is carried out in the study areas of a university to investigate the sound sources, users' reactions, coping methods as well as the perceptual dimensions of the indoor soundscape [10]. Yilmazer and Bora gathered new research results dealing with the indoor soundscape in the metro station including urban soundscape and showed how a built environment affects the pedestrians/passengers and how they perceived their auditory environment. Aletta et al. conducted research in nursing homes, aiming to increase the quality of life of the residents, reduce the behavioral and psychological symptoms of dementia and improving the everyday experience of residents and staff members of the nursing homes [26]. Meng et al. examined the influence of various spatial and environmental factors on subjective loudness and acoustic comfort in five different underground shopping streets [27]. Their results indicate that subjective loudness is influenced by humidity and luminance, while acoustic comfort is influenced by humidity, luminance, and air pressure. They also observed that subjective loudness is higher in street type, shopping streets than square type. Square type shopping streets are also made out to provide more acoustic comfort [27]. Dokmeci, Yorukoglu and Kang tested an indoor soundscape questionnaire which they had developed through pilot studies [28]. This questionnaire focused on the effects of demographical and space usage factors on expectation, perception and reaction of users. They found that expectation is affected by preference, gender and education level.

Soundscape approach is also used to evaluate the acoustic environment of libraries. Mattern in 2007, suggested that we need to consider new ways of thinking about sound in libraries [23]. She proposed that sound should not be considered as something to be eliminated or controlled but rather to be orchestrated and designed for, by considering what sounds people, media and buildings make, as well as using design to promote interaction between them [23]. Xiao and Aletta used soundscape approach to formulate design strategies for achieving acoustic comfort in Birmingham
Library [24]. Their results indicated that users' judgment of the appropriateness and comfort of multifunctional libraries depend on three types of soundscape cognitions which are closely related to the purposes of using the space [24]. They found the layout of the spaces to be of critical importance to acoustic comfort. Dokmeci, Yorukoglu and Kang also used a library as a case study setting to introduce an indoor soundscape framework and explain the variables that contribute to the overall evaluation of an indoor soundscape [22]. They found SPL and loudness are affected by the crowd level as well as the spatial volume and height of the atrium space.

Libraries, in general, have similarities with the setting of this paper, the museums. While both of them are serving as cultural hubs and sources of knowledge for their societies, they are affected by the developments in technology and seeking to find innovative ways of passing this knowledge down to their users/visitors. The traditional method of regulating the sounds people make rules out human perception. The design philosophy of such spaces should consider the interaction of sounds of people, media, building materials, how to determine various spaces as acoustics zones, how these zones should be positioned in relation to one another, and what practices of listening and learning should be promoted in these zones [23].

As can be seen from the literature examples, there are various types of different indoor environments which serve different functions and activities, therefore having completely different sound environments. Requirements emerging from the sound environment of an office are completely different from that of a leisure space as the meanings are given to the sound in context are different. This case is especially true for a museum. While there are museums which are dedicated to a single exhibition type, like a painting museum, there are also museums which have a variety of different exhibitions, each prioritizing a different sort of interaction with the visitors or just having different contexts. In a space like this, the sound environment can be changed in each exhibition area. The museum environment would be an opportunity to use the soundscape approach as a design element which can enhance the visitor experience.

This paper is concerned with the characterization of the acoustic environment, which will be used as a basis for potential future design suggestions. It does not include application or testing of detectable soundscape design suggestions at this point. This characterization procedure comprised of understanding the role of sound in a museum environment, its perception by the users and how it can be improved. The research aimed to do this by combining qualitative and quantitative research methods. Therefore, the study is divided into two parts. Part one is the qualitative half of the study. For this part, semi-structured interviews are conducted with visitors in the museum as part of the Grounded Theory (GT) method, to create a conceptual framework which reflects visitors' interpretation of the indoor soundscape of a museum. This conceptual framework is also used for deriving hypotheses paths for the second part of the research, which is the quantitative half. For this second part, the conceptual framework from the former part is used as a basis for the Structural Equation Modelling (SEM) method [29]. This method used the data gathered from a questionnaire survey to test the explanatory conceptual model of part one, which shows the factors that can potentially affect the interpretation of the indoor soundscape. The second part of this survey focuses on testing the relevance of the categories, as well as the proposed interrelations between them. As the categories of the conceptual model are latent constructs they are observed and measured indirectly. Through SEM, these constructs and their interrelations can be indirectly measured through a series of observable variables (questionnaire responses). By using this multivariate statistical method we can make an inference about the categories of the 
conceptual framework. Therefore, the aim of the SEM is to test the causal relations between each category of the conceptual framework and find out about the direction of the relations and their statistical significance.

\section{Methods}

\subsection{Research setting}

Cengelhan is located at the historic commercial center of Ankara, which is known as the Atpazarı square. Its location is right in front of the main gate of the Ankara Castle. This district is also referred to as the caravanserai district due to a large number of caravanserais [30]. The building was constructed between 1520 and 1521 . It was originally built as a caravanserai, a historic Ottoman/Seljuk building type that combines commerce and accommodation for travelers [31]. Through the centuries, however, it was used for various functions until its restoration and conversion into a museum in 2005. Its front façade and the ground floor of the inner courtyard was occupied with stores, which function as a marketplace/bazaar. During the second half of the twentieth century, however, it was used as a tannery and it was mostly in disrepair. After the restoration, the building and other adjacent caravanserais turned the area into a touristic, cultural hub. The museum houses exhibitions which reflect the industrial and engineering marvels of the last century.

The building shows the typical characteristics of a caravanserai. The two-story building has an inner courtyard and a basement floor. The courtyard is in the middle, surrounded by vaulted cloisters (Fig. 1). The inner courtyard is covered with a glass roof during the restoration (Fig. 1) and has a total height of $10.5 \mathrm{~m}$. Ceiling height of the ground floor is $3.85 \mathrm{~m}$, while the ceiling height of the upper floor is $3.40 \mathrm{~m}$. Courtyard floor is covered with a layer of thin carpet over the original stone floor. Rest of the enclosed space floors are generally covered with a stone finish, but wooden floor planks are also used in some rooms. The cloisters and walls are made out of a combination of brick and local stone. Their ceilings are brick vaults.

In situ measurements of LAeq are only used for acquiring basic information about the acoustical conditions within the museum [32]. Sound levels were measured in the second part of the research, while the questionnaire survey was conducted. The equipment used for the measurement is the Bruel \& Kjaer Sound Level Meter type 2230. Sound levels were measured at different locations (Fig. 1) in the courtyard over fifteen-minute intervals for four hours on each research day, during the visiting hours. According to this, the average sound level $\left(L_{\text {Aeq }}\right)$ is measured as 63.2 ( $\left.L_{\text {Amax }}: 70.5, L_{\text {Amin }}: 57.9\right)$ [29].

\subsection{Data collection and data analyses}

The main body of the research is divided into two as qualitative and quantitative parts. Consequently, data collection is also held through two different methods. Semi-structured interviews are used as the main tool to collect qualitative data for the Grounded Theory (GT) analysis. Afterward, a questionnaire survey is prepared and conducted for the quantitative part (SEM analysis).

\subsubsection{Participants}

Different groups of individuals have participated in each part of the research. A total of 15 individuals, 10 women and 5 men (M age $=34 ; \mathrm{SD}=13.7$ years, age range $24-64$ ) volunteered to be interviewed for the first part of the survey. Majority of these participants were graduate students of Interior Architecture and Environmental Design Department, while the rest were randomly approached visitors. All but one of the participants were native Turkish speakers and of Turkish descent. The remaining participant was Lebanese, therefore the interview was held in English [32].

For the second part of the survey, a sample of 113 visitors, 66 women and 47 men $(\mathrm{M}$ age $=31 ; \mathrm{SD}=12.8$ years, age range 19-67), participated in the questionnaire survey. All of these
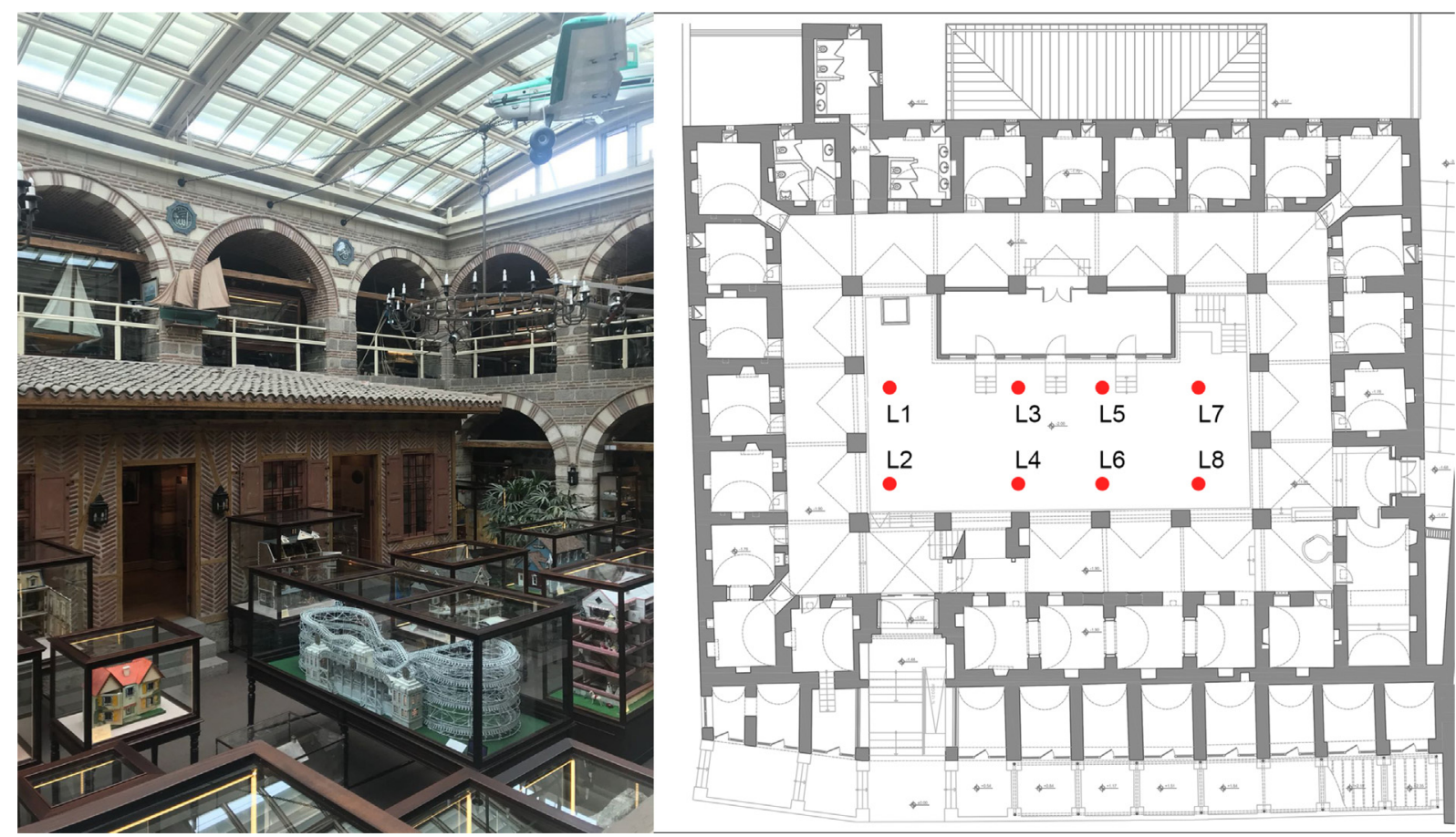

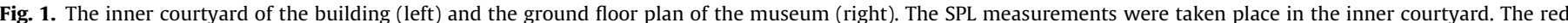

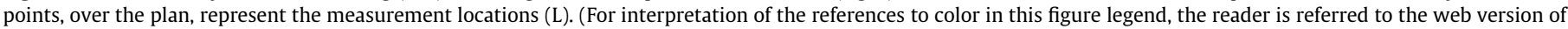
this article.) 
participants were randomly approached museum visitors, who volunteered to participate. They were all native Turkish speakers.

\subsubsection{Grounded theory (GT)}

Grounded Theory (GT) is a user-centered method which is used for systematically analyzing the qualitative data. This method was used also by soundscape researchers previously [8,14-16,20,33] and it can be briefly summarized as " the discovery of theory from data" [34]. The main advantage of GT comes from the fact that it can analyze the qualitative data systematically and traceably while providing in-depth information about the phenomenon [14]. This is achieved through face to face interviews, constant comparison, theoretical saturation, systematic coding, conceptualizing, variation and integration $[14,16,35,36]$.

The main data collection tools are face to face interviews and observation protocols. This method is concerned with the quality of the data samples rather than the quantity. Due to this, insights and depth of information provided by interviewees' subjective evaluation of the acoustic environment are crucial as they are considered as an "ear-witness" [13].

Unlike most other methods, data collection and analysis go hand in hand in GT. This process relies heavily upon two concepts; constant comparison and theoretical sampling. The constant comparison allows the researcher to develop an inductive theory by coding, categorizing and connecting the data, as soon as it is collected. It is the analytical process of comparing the new data with the previously collected, based on similarities and differences. In this process, the researcher looks for similarities and differences between conditions and consequences surrounding key events, incidents and patterns in the data [37].

Constant comparison process includes three phases of coding. The first phase, open coding, separates the data into pieces. The interview transcriptions are analyzed sentence by sentence (Table 1). Each significant event within these sentences is labeled. These labels hold the essence of each significant event. Timonen et al. described this phase as "to break open a topic, after which

\section{Table 1}

Examples from the coding process. The data are broken down into labels and then those labels are put back together through conceptualizing and creating categories.

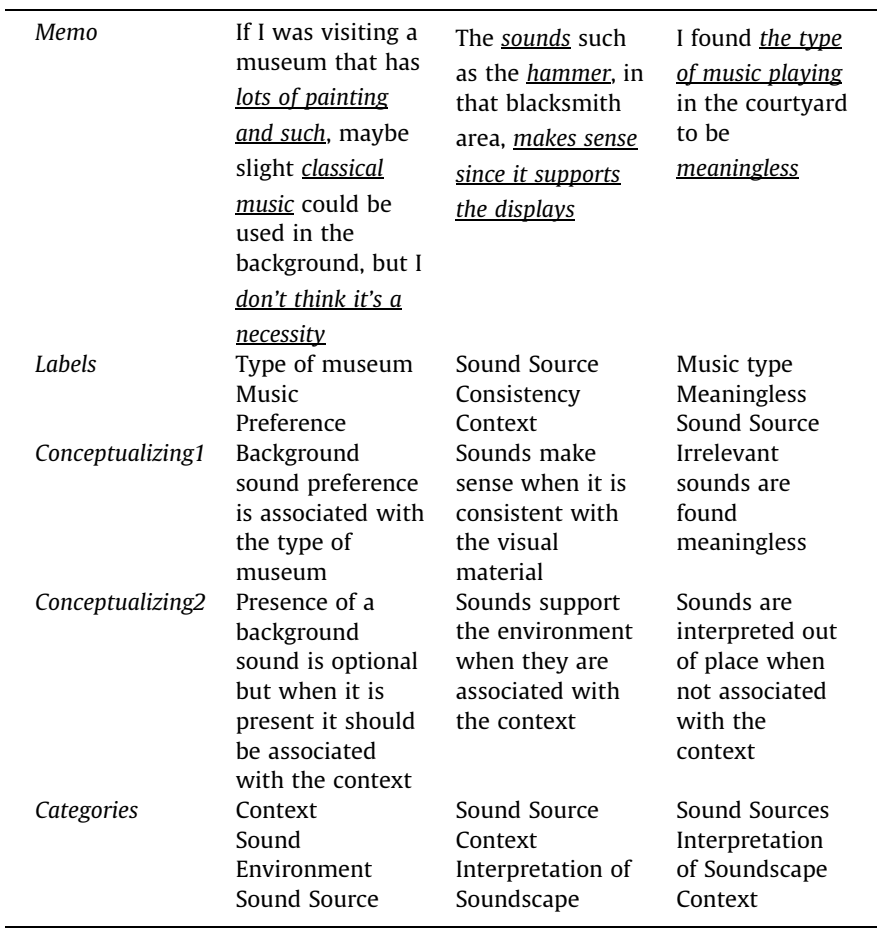

theoretical sampling can be employed more strategically to look for the same code arising elsewhere in the data set" [37].

In the second phase, axial coding procedure is used to put the pieces back together. The labels that are created in the first phase are compared continuously with the newly emerging labels and grouped based on their associations. This creates the initial categories of the emerging conceptual framework. Each category is also compared with each other to find out about any existing associations. Categories are arranged based on these relations and create the conceptual framework. The last phase is called selective coding. In this phase, a category which is central to the phenomenon is selected as the core category. Its relation to other categories is explored which provides a theory regarding the possible explanation of the phenomenon. Atlas.ti 8.3.20 qualitative data analysis software is used for coding the interviews.

Theoretical sampling refers to the process of systematically selecting the participants who will provide data samples that are more likely to contribute to the development of the theory [36]. Theoretical sampling is concerned with the quality of the information provided by the sample rather than the sample quantity, meaning that there is no minimum number of participant requirement $[16,34,36]$. The data samples are chosen in a way that enables the emergence of new categories and further developing the already existing ones effectively. To achieve this, participants are selected from those who can potentially provide answers with a minimal difference and with a maximum difference. Minimizing the differences allows the development of the categories and their properties. Maximizing the differences ensures the saturation of the data samples (theoretical saturation) and the generalizability of the theory [35]. Data collection stops once it reaches theoretical saturation.

For this research, the interviewers consisted of interior architecture graduate students and random museum visitors. Unlike most of the ordinary visitors, the interior architects are more capable of providing a design influenced information based on their expertise on the subject matter. Some elements of the museum might have gone unnoticed by an ordinary visitor, or might be perceived unconsciously. Once it is pointed out by an expert's insight, it can be confirmed by the visitor and other experts, which helps the full development (or grounding) of a category. This ensures the emergence and development of categories throughout the interviews.

The researcher is the limitation of GT and similar narrative interview methods. Whole data collection and analysis process is handled by the same person, which makes him/her part of the process and not value neutral. The researcher needs to be as objective as possible and his/her lack of experience and interviewing skills must not limit the generalizability of the emerging theory $[7,16]$ The researcher must orient towards understanding what is happening, what are the major patterns and what their explanations are and must seek clarification about, how key events, incidents, and behaviors grounded in the data are shaped by context [37].

The interviews are held in the museum café after visitors finish their museum tour and their duration ranged between 9 and $19 \mathrm{~min}$. Semi-structured interviews started with ten core questions (Table 2). It is not uncommon for GT interviews to start off somewhat unstructured as this helps the researcher to maintain openness to the data in the beginning [37]. These questions were meant to be very generic and allowed the researcher to delve deeper into the phenomenon. As the interviews proceeded, participants' significant remarks regarding the phenomenon were added as follow up questions. All interviews were recorded and transcribed verbatim. Data collection continued for three days and it ended once the data reached theoretical saturation and no longer provided any new insight [16,32]. 
Table 2

The ten core questions prepared at the beginning of the semi-structured interviews.

Do you usually go to places like this?

How can you describe the theme of the museum?

What did you think when you first saw this place?

What kind of sounds comes into your mind when thinking about a museum?

What sounds did you hear in the museum?

How can you describe the acoustic environment of this place?

What factors do you think influence the perception of the acoustic environment? [38]

How would you decide if sounds were wanted or unwanted in a soundscape? [38]

Do you think sounds influence your behavior? [38]

How did you feel about this place overall?

\subsubsection{Structural Equation Model (SEM)}

The conceptual framework, created with the Grounded Theory (GT), is used as the basis for the quantitative part of the research. The first part of the research reflected the interpretation of the indoor soundscape and the factors that have potential influences on it. SEM model aims to explore the statistical significances between different categories of the conceptual model.

A questionnaire was prepared to carry out SEM research. Parts of the questionnaire was based on the previous soundscape research $[9,38]$ and the rest is prepared based on the semistructured interviews from the first part. As the questions were required to be associated with the categories of the framework, the researchers went over the interview transcriptions again, highlighted critical issues for each category and formed questions based on these. It can also be said that the transcriptions were reverse-engineered in a way to come up with relevant questions.

The questionnaire consisted of eight different sections, the first part being the demographic information, while the remaining seven are linked to a specific part (category) of the conceptual model. These parts will be discussed in detail in the results section, but they can be briefly listed as; Expectation, Preference, Sound Environment, Built Environment, Context, Interpretation, and Outcomes. For example, Expectation part included questions such as, "This space is how I expected it to be" while the Context part included "I think the sounds that I heard in the exhibition areas are suitable for this museum." The questionnaire used a five- point Likert scale. The response to each question was between, 1 - Strongly Disagree and 5 - Strongly Agree. The data gathered from each of these questions later became the latent variables.

Before starting the SEM analysis, IBM SPSS Statistics Software 21 had been used for the initial analysis of the data. Explanatory factor analysis had been used to eliminate the questions with low factor loadings or those that did not fit in with the rest of the items in the factor. During this, the researchers also saw that the data was not normally distributed, so it either required to be transformed, or a non-parametric analysis method was required. With this regard, the researchers decided to use the Partial Least Squares Structural Equation Model (PLS-SEM). This method is more suitable then regular SEM when the data distribution is nonnormal, the sample size is relatively small and when the nature of the study is an explanatory one [39]. Given the fact that the research has all of these aspects, the PLS-SEM method was preferred over the SEM. Smart PLS 3 was used for the data analysis. The latent variable structural model was used to evaluate the relationships between the categories of the framework.

\section{Results}

\subsection{Grounded theory}

Seven categories were generated at the end of the data analysis, which are Sound Environment, Built Environment, Context, Preference, Expectation, Interpretation of Soundscape and Outcomes (Table 3). Categories were arranged in a graphical order based on the associations between them. This work created a conceptual framework which reflected the factors that influence visitors' interpretation of the indoor soundscape of a museum (Fig. 2). Elements of this framework will be explained in the following sections.

\subsubsection{Expectation and Preference}

Expectation and Preference can be referred to as the causal conditions of the framework [32]. Based on the interviews, it was seen that once visitors had entered the museum, they subconsciously

Table 3

Core and sub-categories of the conceptual framework [32].

\begin{tabular}{|c|c|c|c|c|c|c|}
\hline Expectation & Preference & Built Environment & Sound Environment & Context & Interpretation of Soundscape & Outcomes \\
\hline & & $\begin{array}{l}\text { - Physical Aspects } \\
\text { - Perceptual Aspects }\end{array}$ & - Sound Sources & $\begin{array}{l}\text { - Consistency } \\
\text { - Inconsistency }\end{array}$ & $\begin{array}{l}\text { - Positive Interpretation } \\
\text { - Neutral } \\
\text { - Negative Interpretation }\end{array}$ & \\
\hline
\end{tabular}

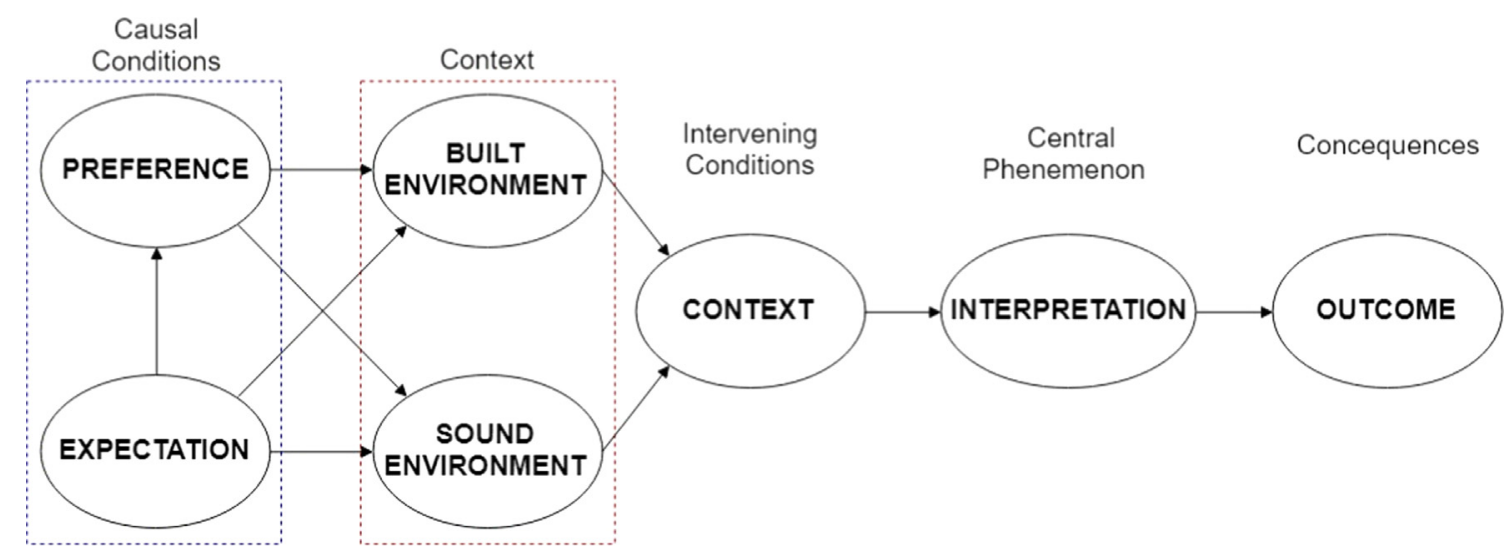

Fig. 2. The conceptual framework which is created at the end of part one. 
decided whether the environment was up to their expectations and preferences.

Almost half of the interviewed participants had never visited the museum beforehand they were completely unaware of the theme of the museum. Some of the participants were professional architects who had knowledge about the necessities of museum design and had very fixed expectations about the subject. Majority of the participants' expectations were related to the built environment rather than the sound environment.

Expectation also has influences on individuals' preferences. Both expectations and preferences are predetermined and formed by prior knowledge. Majority of the individuals preferred to have a quiet environment in the museum as they expected that it would be hard to concentrate on the exhibit if the environment was loud. Similarly, some of them preferred the museum to be in a modern building, but they said they were satisfied with their visit regardless of their preferences before visiting it. Based on this, it can be said that Expectation and Preference are the first factors that influence the interpretation of the soundscape and Expectation also has an influence over Preference up to a certain degree.

\subsubsection{Sound Environment and Built Environment}

Sound Environment and Built Environment can be referred to as the context of the research. They fit this role taking the qualitative research terminology into account and they should not be confused with the context of the sound. They refer to the location, in which the actions are taking place.

Based on data analysis, the category of Built Environment is divided into two subcategories (Table 3), Physical Aspects of the Built Environment and the Perceptual Aspects of the Built Environment. Physical aspects of the Built Environment comprised of visitors' comments regarding materials, layout, the content of the exhibition and their descriptions of the space (large-small, openenclosed, historical- modern). On the other hand, perceptual aspects of the space comprised of statements that describe how they perceived the space, regardless of the sound environment, such as, cozy, orderly-complicated, crowded and boring/ordinary. Visitors' experiences form Sound Environment regarding the sound environment (high-low sound levels, echo, and controlleduncontrolled sound environment) and it only has one subcategory, which is sound sources.

\subsubsection{Context}

Context is the intervening condition of the conceptual framework. Context shapes and/or constrains the interpretation of the sound environment. Context is divided into two as consistency and inconsistency. In this framework, the main role of context is its consistency with the environment. Throughout the research, it has been observed that as long as it is consistent with the context even the most frustrating sound environments can be interpreted at least non-irritating, if not satisfactory. A clear example of this is observed in the toys section of the museum. This section is dedicated to old toys, bicycles, doll houses, etc. Some of the interviewees visited this section of the museum while a large group of elementary school student was getting around. Even though the school children made the environment feel loud and crowded, various accounts stated that they did not find the sound of the children annoying or disturbing. When asked about this event, one participant $(P)$ said:

P: That part, downstairs, was the toys section. Children were yelling at each other, some of them were calling for their teacher. It matched what was displayed. What I was looking at was kids' stuff. Sound matched with the objects and created strong interaction (translated from Turkish) [32].

\subsubsection{Interpretation of the soundscape}

Central phenomenon of this research is the Interpretation of the Soundscape [32]. Its subcategories consist of positive, negative and neutral interpretation of the soundscape. These subcategories are also a response to the interpretation of the soundscape. When the interpretation is positive, it can lead to the appreciable, interesting or intriguing environment. On the other hand, when it is negative, it leads to an irritating, unenjoyable environment.

The context of the sound is the major factor that influences the interpretation of the sound. As it was demonstrated through visitor quotes soundscape can be interpreted positively if it matches with individuals' expectations of context. This interpretation does not mean that positive interpretation occurs only when space is consistent with individuals' expectations, as the expectations do not have to be always positive. An individual might not be very interested in visiting a museum, expect it to be an ordinary and boring environment and can still be impressed at the end of his/her visit.

Even though having a background sound was appreciated by the majority of the participants, almost all expressed dissatisfaction with the type of music that was being broadcasted. They thought that the current music of choice was very irrelevant and made it harder for them to concentrate. Participants desired background to be classical music, as they felt that it would not interfere with their concentration, while still creating a soft background sound. Almost every interviewee agreed that having a background sound was much preferable to the absence of it. They were aware that it is impossible to rid the space of uncontrolled sounds and having a background sound works as a masking noise up to a certain degree. Among all, human-generated sounds were found to be the most disturbing and negatively interpreted sounds.

\subsubsection{Outcomes}

Outcomes are the consequences of the responses that are given to the interpretation of the sound environment. A soundscape that is interpreted as interesting, intriguing or apparent can result in a positive contribution to the atmosphere of the space. The most solid outcome of a positively interpreted soundscape for this space is the sense of increased interaction with the displayed objects, which also promotes a considerable museum experience. If one considers the fact that it is not usually allowed to touch the displayed objects, the best way of forming an interaction with the exhibit is by hearing it.

Each section of the museum is dedicated to a specific theme. Some of these themes are craftsmanship, historic cars, vintage toys, and engineering marvels. To some extent, the sound is used as a design element to create the feeling of being present in that environment. Buildings' historic characteristic greatly helped participants to imagine themselves being in the actual environment. This atmosphere is further enhanced with the introduction of different sounds depending on the contents of the exhibitions. In the displays, like of blacksmithing or carpentry, hearing the sound of the hammer hitting the anvil or a saw cutting through a wood plank made visitors imagine themselves traveling through a bazaar of old times. This experience is further enhanced with the historic character of the building. In a way, it can be said that proper usage of sound gave life to space.

P: It was like the museum was blended into real life. I felt like I toured through a bazaar rather than a museum [32].

The combination of the historical aspect, function and the sound of the building creates a unique atmosphere. As a participant stated, the mixture of the historic stone walls and the wooden floors, with the exhibitions that display the life of the past generations create a visual atmosphere that takes the participants dec- 
ades back in time. Addition of proper sound elements further enhances the atmosphere and creates a unique sensation that feels like the building is speaking to them.

P: It felt like I went back in time and lived that moment. It was not just a space surrounded by four walls. It had a unique atmosphere [32].

Every participant appreciated the usage of sound based on the theme of the section. However, some of them complained that sound levels were too high in some sections and too low in some others which caused sound interference. Participants especially enjoyed the sections where they can control the sound with buttons, but could not use this to its full extent as the sound within that room was too low while the sound outside the room was too high. This created a sense of frustration as the participants' tried to understand the message conveyed through the sound.

As it was expressed earlier, under the right context even the most frustrating sound sources, like the sound of yelling children, can be appreciated. But, when these children moved from the toys part to a more serious section, their uncontrolled sound became an issue. Participants expressed that when space became so crowded and filled with irrelevant speech, it caused a loss of concentration, disturbance and made them want to get away from that part of the exhibition as an outcome.

\subsection{Structural equation model}

\subsubsection{Confirmatory factor analysis}

The data gathered from the questionnaire survey was analyzed with IBM SPSS Statistics Software 21 and Smart PLS software. Explanatory factor analysis was conducted as a means of preprocessing the data. Principle Component Analysis method was used with Varimax rotation to see the factor loading of each indicator and also to see if the indicators formed proper factors in their relevant categories. The proposed structural model is a reflective model, therefore, indicators with low factor ratings are eliminated during this stage. The Kaiser-Mayer-Olkin (KMO) test yielded a score of 0.733 , which indicates that the sample group is suitable for factor analysis. Once the preliminary analysis was done, Smart PLS software was used for preparing the structural model.

Confirmatory factor analysis was used for examining the reliability and validity measures. Summary of these measurements can be seen in Table 4. As this is a reflective model, Composite Reliability, Cronbach's Alpha and AVE (Average Variance Extracted) scores were utilized to examine the construct validity and reliability of the model [39]. The AVE (Average Variance Extracted) ratings are all between 0.500 and 0.676 which meant that all indicators were right above the cut off value of 0.05 [40]. All composite reliability values were also above the required cut off value of 0.70 , ranging between 0.751 and 0.853 . On the other hand, some of the Cronbach's alpha values were lower than the cut off value of 0.70 . Considering that this research is focused on an explanatory model and two of the Cronbach's values are very close to the cut off value of 0.70 , it can be said that model has adequate construct validity and convergent validity.

\subsubsection{The Structural Model (SEM)}

Fig. 3 shows the results of the latent variable path model. As can be seen from Table 5 , five paths out of nine reached statistical significance. The first hypothesis indicated that Expectation has a positive association with Preference. Regarding the first hypothesis (H1a), it was found that the association between the Expectation and Preference are statistically significant. This result confirms that expectations do have an effect on preferences, but this effect has a low positive path coefficient (0.288). Rest of the hypotheses regarding Expectation (H1b and $\mathrm{H} 1 \mathrm{c}$ ) are not statistically significant. These hypotheses stated that Expectation could have a positive association with the Built Environment (H1b) and the Sound Environment (H1c). The structural model did not support these hypotheses, specifically, the $\mathrm{H} 1 \mathrm{~b}$ has a very low $\mathrm{t}$ score $(t=0.262)$ and a very high $p$-value $(p=0.793)$. Both of them also have low path coefficients.

Similarly, no statistical significance was observed for any hypotheses regarding Preference. Preference did not have a statistical significant effect on the Built Environment (H2a) or the Sound Environment (H2b). Both of them also have very low path coeffi-

Table 4

Factor loadings, construct validity and convergent validity score of the proposed model.

\begin{tabular}{|c|c|c|c|c|c|}
\hline Factor & Indicator & Factor Loading & Cronbach's Alpha & Composite Reliability & AVE \\
\hline Expectation & $\begin{array}{l}\text { EX1 } \\
\text { EX2 } \\
\text { EX3 }\end{array}$ & $\begin{array}{l}0.818 \\
0.730 \\
0.543\end{array}$ & 0.524 & 0.744 & 0.500 \\
\hline Preference & $\begin{array}{l}\text { PRF1 } \\
\text { PRF3 } \\
\text { PRF4 }\end{array}$ & $\begin{array}{l}0.850 \\
0.927 \\
0.531\end{array}$ & 0.722 & 0.824 & 0.621 \\
\hline Sound Environment & $\begin{array}{l}\text { SE1 } \\
\text { SE4 } \\
\text { SE5 }\end{array}$ & $\begin{array}{l}0.803 \\
0.616 \\
0.735\end{array}$ & 0.544 & 0.764 & 0.522 \\
\hline Built Environment & $\begin{array}{l}\text { BE4 } \\
\text { BE5 } \\
\text { BE6 } \\
\text { BE8 }\end{array}$ & $\begin{array}{l}0.605 \\
0.798 \\
0.823 \\
0.707\end{array}$ & 0.714 & 0.825 & 0.545 \\
\hline Context & $\begin{array}{l}\mathrm{CO} 1 \\
\mathrm{CO} 2 \\
\mathrm{CO} 3\end{array}$ & $\begin{array}{l}0.813 \\
0.859 \\
0.669\end{array}$ & 0.693 & 0.825 & 0.615 \\
\hline Interpretation & $\begin{array}{l}\text { INT1 } \\
\text { INT4 } \\
\text { INT5 }\end{array}$ & $\begin{array}{l}0.699 \\
0.880 \\
0.874\end{array}$ & 0.754 & 0.861 & 0.676 \\
\hline Outcomes & $\begin{array}{l}\text { OUT1 } \\
\text { OUT3 } \\
\text { OUT4 } \\
\text { OUT5 }\end{array}$ & $\begin{array}{l}0.690 \\
0.675 \\
0.837 \\
0.676\end{array}$ & 0.695 & 0.813 & 0.523 \\
\hline
\end{tabular}




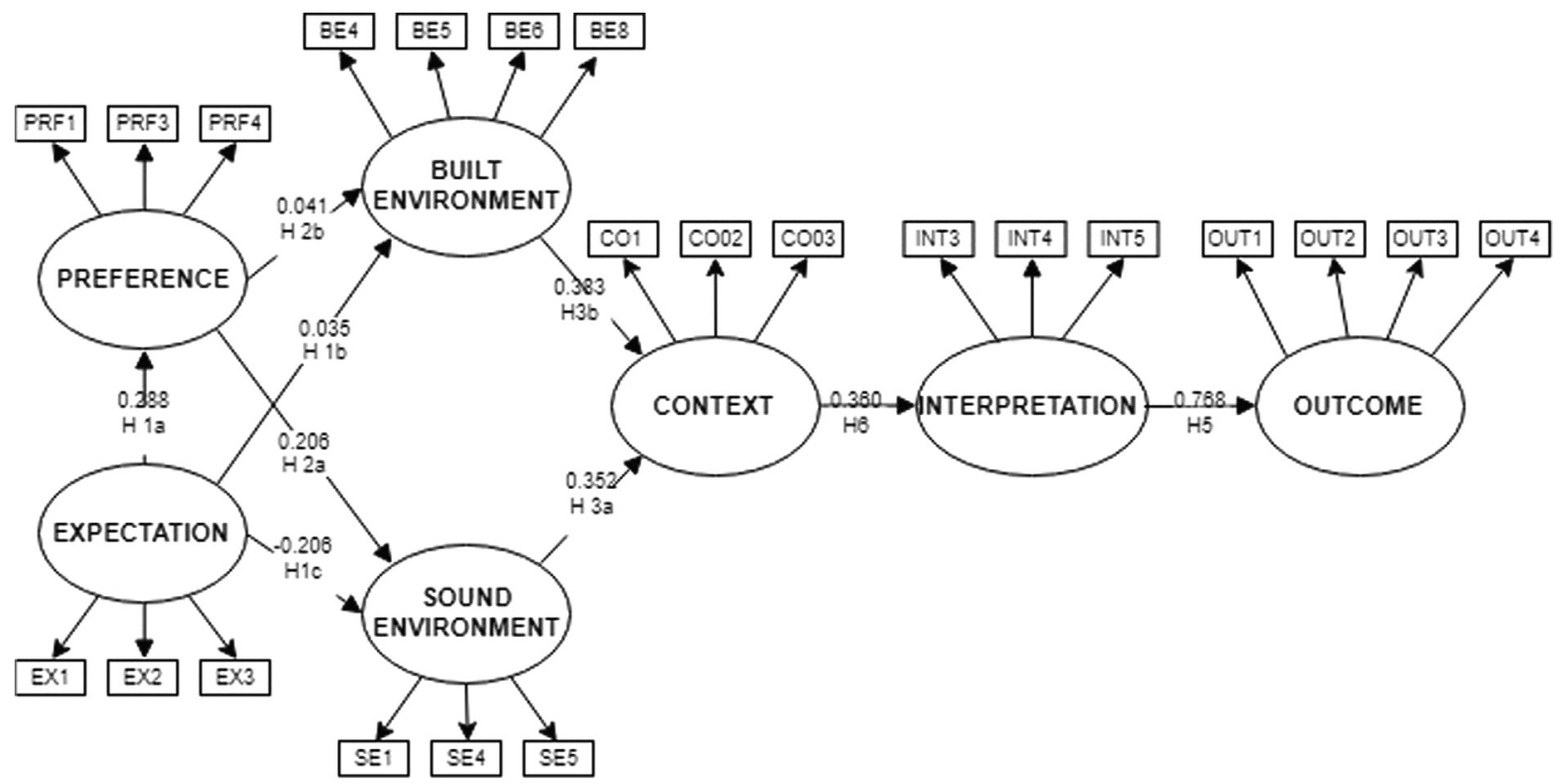

Fig. 3. The structural equation model with latent variables and path coefficients. The label for each hypothesis can be found under the path coefficient.

Table 5

Results of the hypothesis testing ( ${ }^{*} \mathrm{p}<0.05,{ }^{* *} \mathrm{p}<0.01$.).

\begin{tabular}{|c|c|c|c|c|c|}
\hline Factor Pairs & Hypothesis Path & Sample Mean (M) & Standard Deviation (S.D.) & T Statistics & P Values \\
\hline Expectation - Preference & H1a & 0.302 & 0.104 & 2.757 & $0.006^{* *}$ \\
\hline Expectation - Built Environment & $\mathrm{H} 1 \mathrm{~b}$ & 0.015 & 0.134 & 0.262 & 0.793 \\
\hline Expectation - Sound Environment & $\mathrm{H} 1 \mathrm{c}$ & -0.213 & 0.121 & 1.702 & 0.089 \\
\hline Preference - Built Environment & $\mathrm{H} 2 \mathrm{a}$ & 0.054 & 0.125 & 0.325 & 0.746 \\
\hline Preference - Sound Environment & $\mathrm{H} 2 \mathrm{c}$ & 0.110 & 0.110 & 1.871 & 0.062 \\
\hline Built Environment - Context & H3a & 0.389 & 0.100 & 3.849 & $0.000^{* *}$ \\
\hline Sound Environment - Context & $\mathrm{H} 3 \mathrm{~b}$ & 0.369 & 0.098 & 3.609 & $0.000 * *$ \\
\hline Context - Interpretation & $\mathrm{H} 4$ & 0.468 & 0.079 & 5.717 & $0.000^{* *}$ \\
\hline Interpretation - Outcomes & H5 & 0.770 & 0.049 & 15.676 & $0.000^{* *}$ \\
\hline
\end{tabular}

cients, in fact, the coefficient between preference and built environment is the lowest among all. This analysis showed that there is no association at all (Fig. 3).

Both the Sound Environment and Built Environment are statistically significantly associated with Context. Path analysis shows that there is a low to the medium positive association between the Built Environment and the Context (H3a). This analysis indicates that as users' satisfaction with the built environment increases they find the environment more consistent with the context. Similarly, there is a low to the medium positive association between the sound environment and the context ( $\mathrm{H} 3 \mathrm{~b})$, indicating that as users' satisfaction with the sound environment increases, they find the sound environment consistent with the context.

There is a statistically significant relationship between the Context and the Interpretation of the Soundscape (H4). According to the path coefficients, the direction of this relationship is positive. This analysis shows that indoor soundscape is interpreted more positively when it is consistent with the context. It should also be noted that the Built Environment and Sound Environment categories are indirectly affecting the Interpretation of the Soundscape, as they are associated with the Context in a statistically significant manner. This also means that Context acts as a mediator between the Sound and Built Environment and the Interpretation of Soundscape. This supports the soundscape literature, which places context as the key/critical factor in terms of interpreting the soundscape.

The last hypothesis is about the Interpretation of the Soundscape and the Outcomes of this interpretation (H5). Highest statistical sig- nificant and path coefficient was observed for this hypothesis (path coefficient: $0.768, t=15.676, p<0.000)$. According to this, there is a statistically significant high amount of positive association between the interpretation of soundscape and the outcomes. This result can be explained as the interpretation becomes more positive or satisfactory, outcomes of this interpretation also become more positive. For this case, positive interpretation subcategories are, pleasing, appreciation, satisfaction, intriguing, calming and the positive outcomes were the enhanced atmosphere, feeling of connection with the environment, promoting focus and space experience. Visitors' museum experience was positively affected as they found the soundscape more intriguing, pleasing, etc. Similarly, if they interpret the soundscape negatively, such as distracting or unenjoyable, they feel the urge to end their tour end leave the space or at least that certain part of the exhibition.

\section{Discussion}

This study aimed to use qualitative and quantitative methods to develop a conceptual framework, create hypotheses based on this framework and test them using the Structural Equation Modelling (SEM) approach. Most of the categories obtained from the conceptual framework were similar to the previous qualitative research [15,16,33,41] and the ISO 12913-1 [5]. Similar to those, it was found that context is the most crucial element towards the interpretation of the soundscape. In this sense, the context of the sound is not independent of the physical environment [8]. 
Some unexpected findings were obtained from the SEM approach. These surprising findings are mainly related to the Expectation and Preference categories. Results indicate that Expectation and Preference are not statistically significantly associated with neither Built nor Sound Environment. This result is not consistent with the literature [9,33]. Bruce and Davies [9] found that expectations were affecting the soundscape perception by influencing individuals' behaviors and evaluation of the soundscape.

A possible cause of this can be the low Cronbach's alpha value of Expectation (0.524). If this is the case, questions associated with that factors can be redesigned to increase the reliability scores. This result could also be a consequence of the limitations caused by the application of SEM in this research. SEM approach usually requires a fully developed theory with a well-established questionnaire, such as the examples suggested by the ISO 12913-2 [6]. However, as this research focuses on theory building, and needed to develop its own questionnaire to do so, this aspect of SEM is a limitation. We tried to minimize the effect of this limitation by adopting the more flexible and explanatory approach of PLS-SEM. Further study with a more developed questionnaire can improve the results of the model. One possible way which might yield better results for this model is asking a single question about familiarity under the Expectation category, rather than a series of questions about expectation. Considering that the expectations are based on past experiences [9], a first time visitors' expectation will only be based on their visits to similar places but it will still be different from someone who is familiar with the place. This will also change the "Expectation" from a latent variable into an observed variable. Model fit is not discussed in this paper as there is no global goodness of fit measure for PLS-SEM, unlike the covariance-based SEM [39].

Other than the first two categories, the conceptual framework supports the existing literature, which emphasizes the importance of context towards the interpretation of soundscape $[5,42]$. The conceptual framework, developed by the ISO 12913-1, showed the association between context, interpretation of auditory sensation, responses, and outcomes [5]. The most reoccurring theme during the first part of the study was sounds' consistency with the context. The soundscape indicators were not specifically examined, which were briefly mentioned in the introduction section, but it can be said that appropriateness is the most critical soundscape indicator for this research setting. Axelsson [43], along with Xiao and Aletta [24] indicated appropriateness as an important indicator. A sound can be appropriate to a context even though it is poor, which made Axelsson [43] question the usefulness of this indicator. A perfect example of this is observed in the toys section of the museum, in which the sounds of groups of children were poor in quality but appropriate for the context and created less annoyance then one would have thought. But this does not mean that the soundscape quality is fine as it is.

Other indicators, such as pleasantness, also came up, but appropriateness was a significantly more common concern for the visitors. In the model presented in this study, responses are represented with three subcategories under Interpretation, which are: positive, negative and neutral interpretation. These subcategories also have a variety of items under them such as pleasantness, calmness, appropriateness, annoyance, etc.

The indoor environment of a museum can comprise of different sections with various themes. Physical and auditory elements within the environment create a context. If this context of sound is consistent with the environment or suitable for its function, it can significantly influence the interpretation of the soundscape. One of the key findings found with this research is that the visitors desire the sound environment to be well-designed. When it is consistent with the context, a sound element can improve visitors' interaction with the exhibitions by enhancing the atmosphere.
An example of this was visible on the exhibition area referred to as the "craftsman's street", in which every room of the aisle was a craftsman shop, such as blacksmith, tinsmith, carpenter, etc. This finding is also very historically accurate as this part of the caravanserai was occupied with this kind of shops a century ago. The historic building and the exhibition are already compatible with each other, but without the right sound complementing this, the whole exhibition might feel fake. At the moment, this part of the museum is the only place where we can say that the sound is appropriate for the context and it is also one of the more memorable, enjoyable and authentic parts according to the visitors.

On a busy day, however, no matter how good the sound environment was designed it will be suppressed by the crowd. One possible suggestion is using earphones. This tool may sound like isolating the visitor from the sound environment altogether, but audio tours are commonly used in many museums. Audio tours mostly consist of a narrator explaining the artifacts. Relevant sound compositions can be added to these audio tours or take the storytelling to a new level. Machine learning algorithms can be used to predict visitors' expectations, preferences and attitudes while guiding them through one of the pre-determined paths to travel in the museum. Once enough data for artificial intelligence (AI) is gathered, this can also be used as a design tool rather than a guidance tool.

The historic building's contribution to the overall experience should not be overlooked. Therefore, some of the findings may not be generalizable in a similar manner to all museum types, such as for a modern art museum in a deconstructivist building. One thing that is certain is the importance of the appropriateness of the sound to the context. The output of the interpretation may change, but the interpretation itself will be the same. If the sound environment is interpreted positively it may not necessarily increase interaction but promote concentration.

\section{Conclusion}

This research aimed to use qualitative and quantitative methods to develop a conceptual framework, create hypotheses based on this framework and test them using the structural equation modelling (SEM) approach. The conceptual framework showed that meanings associated with a sound in context are the major element that contributes to the interpretation of the context. This also meant that appropriateness is a vital soundscape indicator, which should be highly considered when designing a museum.

Out of the nine hypotheses that were derived from the conceptual framework, five of them showed statistical significance. The ones that did not show significance were all related to the Expectation and Preference categories. This was a surprising finding as it was inconsistent with the literature. This might be caused by a relatively low-reliability score of the questions regarding Expectation. Through further study and modification of the questionnaire, the results can be more clearly understood and improved.

Findings indicated the individuals' desire for a well-designed sound environment just like the physical environment. Under right conditions, sound can be used to create a living environment with a unique atmosphere that would enhance the visitors' experience to a maximum degree.

\section{Acknowledgments}

We appreciate the graduate students of I.D. Bilkent University, Department of Interior Architecture and Environmental Design for their support. This research is conducted as part of a Ph.D. study in I.D. Bilkent University. We also would like to thank Cemre Orhan for her contributions during data collection and analysis. 


\section{References}

[1] International Council of Museums. ICOM code of ethics for museums; 2017. p. 15.

[2] Dal Falco F, Vassos S. Museum experience design: a modern storytelling methodology. Des J 2017;20:S3975-83. https://doi.org/10.1080/ 14606925.2017.1352900.

[3] Shaw C, Ivens J. Building great customer experiences. New York: Palgrave Macmillan; 2002. , https://doi.org/10.1057/palgrave.im.4340221.

[4] Huang L, Kang J. The sound environment and soundscape preservation in historic city centres-the case study of Lhasa. Environ Plan B-Plan Des 2015;42:652-74. https://doi.org/10.1068/b130073p.

[5] International Organization for Standardization. ISO 12913-1 AcousticsSoundscape-Part 1: Definition and conceptual framework. Geneva: ISO; 2014

[6] International Organization for Standardization. SO/TS 12913-2:2018 Acoustics-Soundscape-Part 2: Data collection and reporting requirements. Geneva: ISO; 2018.

[7] Aletta F, Kang J, Axelsson Ö. Soundscape descriptors and a conceptual framework for developing predictive soundscape models. Landsc Urban Plan 2016;149:65-74. https://doi.org/10.1016/i.landurbplan.2016.02.001.

[8] Mackrill J, Cain R, Jennings P. Experiencing the hospital ward soundscape: towards a model. J Environ Psychol 2013;36:1-8. https://doi.org/10.1016/ i.jenvp.2013.06.004.

[9] Bruce NS, Davies WJ. The effects of expectation on the perception of soundscapes. Appl Acoust 2014;85:1-11. https://doi.org/10.1016/i. apacoust.2014.03.016.

[10] Acun V, Yilmazer S. Understanding the indoor soundscape of study areas in terms of users' satisfaction, coping methods and perceptual dimensions. Noise Control Eng J 2018;66.

[11] Axelsson Ö, Nilsson ME, Berglund B. A principal components model of soundscape perception. J Acoust Soc Am 2010;128:2836-46. https://doi.org/ $10.1121 / 1.3493436$.

[12] Cain R, Jennings P, Poxon J. The development and application of the emotional dimensions of a soundscape. Appl Acoust 2013;74:232-9. https://doi.org/ 10.1016/i.apacoust.2011.11.006.

[13] Schulte-fortkamp B, Fiebig A. Soundscape analysis in a residential area: an evaluation of noise and people's mind. Acta Acust United Acust 2006;92:875-80.

[14] Fiebig A, Schulte-fortkamp B, Akustik T, Berlin D, Akustik T, Berlin D, et al. The importance of the grounded theory with respect to soundscape evaluation 2004. p. 349-50.

[15] Yilmazer S, Acun V. A grounded theory approach to assess indoor soundscape in historic religious spaces of Anatolian culture: a case study on Hacı Bayram Mosque. Build Acoust 2018. https://doi.org/10.1177/1351010X18763915.

[16] Acun V, Yilmazer S. A grounded theory approach to investigate the perceived soundscape of open-plan offices. Appl Acoust 2018;131:28-37. https://doi. org/10.1016/i.apacoust.2017.09.018.

[17] Davies WJ. Special issue: applied soundscapes. Appl Acoust 2013;74:223. https://doi.org/10.1016/j.apacoust.2012.07.004.

[18] Maristany A, López MR, Rivera CA. Soundscape quality analysis by fuzzy logic: a field study in Cordoba, Argentina. Appl Acoust 2016;111:106-15. https://doi. org/10.1016/j.apacoust.2016.04.013.

[19] Aletta F, Astolfi A. Soundscapes of buildings and built environments. Build Acoust 2018;25:195-7. https://doi.org/10.1177/1351010x18793279.

[20] Cankaya S, Yilmazer S. The effect of soundscape on the students' perception in the high school environment. In: InterNoise 2016. Hamburg: Institute of Noise Control Engineering; 2016. p. 139-46.

[21] Aletta F, Botteldooren D, Thomas P, Devos P, Van de Velde D, De Vriendt P, et al. Monitoring sound levels and soundscape quality in the living rooms of nursing homes: a case study in Flanders (Belgium). Appl Sci 2017;7:874. https://doi.org/10.3390/app7090874.
[22] Dokmeci Yorukoglu PN, Kang J. Analysing sound environment and architectural characteristics of libraries through indoor soundscape framework. Arch Acoust 2016;41:203-12. https://doi.org/10.1515/aoa-2016$\underline{0020}$.

[23] Mattern S. Resonant texts: sounds of the American public library. Senses Soc 2007;2:277-302.

[24] Xiao J, Aletta F. A soundscape approach to exploring design strategies for acoustic comfort in modern public libraries: a case study of the Library of Birmingham. Noise Mapp 2016;3:264-73. https://doi.org/10.1515/noise2016-0018.

[25] Yilmazer S, Bora Z. Understanding the indoor soundscape in public transport spaces: a case study in Akköprü metro station, Ankara. Build Acoust 2017;24:325-39. https://doi.org/10.1177/1351010X17741742.

[26] Aletta F, Botteldooren D, Thomas P, Vander Mynsbrugge T, De Vriendt P, Van De Velde D, Devos P, et al. Exploring the soundscape quality of five nursing homes in Flanders (Belgium): preliminary results from the AcustiCare project. In: Inter-Noise 2017, Hong Kong. p. 31-40.

[27] Meng Q Kang J, Jin H. Field study on the influence of spatial and environmental characteristics on the evaluation of subjective loudness and acoustic comfort in underground shopping streets. Appl Acoust 2013;74:1001-9. https://doi.org/10.1016/i.apacoust.2013.02.003.

[28] Dokmeci Yorukoglu PN, Kang J. Development and testing of indoor soundscape questionnaire for evaluating contextual experience in public spaces. Build Acoust 2017;24:307-24. https://doi.org/10.1177/1351010X17743642.

[29] Yilmazer S, Acun V. A structural equation modelling approach for indoor soundscape: adaptive re-use in Çengelhan Caravanserai. AESOP 18 Gothenburg, 2018.

[30] Ergenç Ö. XVI. Yüzyılda Ankara Ve Konya: Osmanlı Klasik Dönemi Kent Tarihçiliğine Katkı. Ankara: Ankara Ensitüsü Vakfı; 1995.

[31] Caravanserais: cross-roads of commerce and culture along the Silk Roads | SILK ROADS; n.d. https://en.unesco.org/silkroad/content/caravanserais-crossroads-commerce-and-culture-along-silk-roads [accessed December 17, 2018].

[32] Acun V, Yilmazer S, Orhan C. Indoor soundscape of historical spaces: the case of Çengelhan Caravanserai. Euronoise 2018, Crete, 2018.

[33] Liu F, Kang J. A grounded theory approach to the subjective understanding of urban soundscape in Sheffield. Cities 2016;50:28-39. https://doi.org/10.1016/ i.cities.2015.08.002.

[34] Glaser B, Strauss A, Strutzel E. The discovery of grounded theory; strategies for qualitative research. Nurs Res 1968.

[35] Glaser B. Theoretical sensitivity: advances in the methodology of grounded theory. Sociology Press; 1978.

[36] Strauss A, Corbin J. Basics of qualitative research: techniques and procedures for developing grounded theory; 1998.

[37] Timonen V, Foley G, Conlon C. Challenges when using grounded theory 160940691875808. Int J Qual Methods 2018;17. https://doi.org/10.1177/ 1609406918758086.

[38] Davies WJ, Adams MD, Bruce NS, Cain R, Carlyle A, Cusack P, et al. Perception of soundscapes: an interdisciplinary approach. Appl Acoust 2013;74:224-31. https://doi.org/10.1016/i.apacoust.2012.05.010.

[39] Garson GD. Partial least squares: regression \& structural equation models. Asheboro (NC): Statistical Associates Publishers; 2016.

[40] Kline RB. Principles and practice of structural equation modeling, vol. 156; 2011. https://doi.org/10.1038/156278a0.

[41] Mackrill J, Jennings P, Cain R. Exploring positive hospital ward soundscape interventions. Appl Ergon 2014;45:1454-60. https://doi.org/10.1016/i. apergo.2014.04.005.

[42] Kang J, Aletta F, Gjestland TT, Brown LA, Botteldooren D, Schulte-fortkamp B, et al. Ten questions on the soundscapes of the built environment. Build Environ 2016;108:284-94. https://doi.org/10.1016/j.buildenv.2016.08.011.

[43] Axelsson Ö. How to measure soundscape quality. In: Euronoise 2015. p. $1477-81$. 